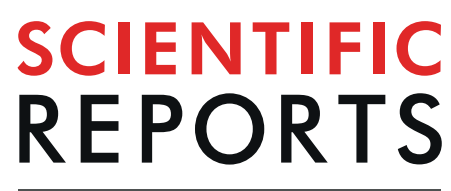

natureresearch

Received: 23 April 2019

Accepted: 10 September 2019

Published online: 10 October 2019

\section{Disruption of $h m g A$ by DNA Duplication is Responsible for Hyperpigmentation in a Vibrio anguillarum Strain}

Veronica Batallones, Jennifer Fernandez, Brett Farthing, Jordan Shoemaker, Keizen Li Qian, Kimberly Phan, Eric Fung, Ashley Rivera, Kevin Van, Francesca de la Cruz, Alexandra J. Ferreri, Krystle Burinski, Jackie Zhang, Vicente Lizarraga, Kevin Doan, Kenneth Rocha, German Traglia, Maria S. Ramirez (D) \& Marcelo E. Tolmasky

Vibrio anguillarum 531A, isolated from a diseased fish in the Atlantic Ocean, is a mixture composed of about 95 and $5 \%$ of highly pigmented cells (strain 531Ad) and cells with normal levels of pigmentation (strain 531Ac), respectively. Analysis of the $V$. anguillarum 531Ad DNA region encompassing genes involved in the tyrosine metabolism showed a 410-bp duplication within the $h m g A$ gene that results in a frameshift and early termination of translation of the homogentisate 1,2-dioxygenase. We hypothesized that this mutation results in accumulation of homogentisate that is oxidized and polymerized to produce pyomelanin. Introduction in E. coli of recombinant clones carrying the $V$. anguillarum $h p p D$ (4-hydroxyphenylpyruvate-dioxygenase), and a mutated $h m g A$ produced brown colored colonies. Complementation with a recombinant clone harboring $h m g A$ restored the original color to the colonies confirming that in the absence of homogentisate 1,2-dioxygenase the intermediary in tyrosine catabolism homogentisate accumulates and undergoes nonenzymatic oxidation and polymerization resulting in high amounts of the brown pigment. Whole-genome sequence analysis showed that $V$. anguillarum $531 \mathrm{Ac}$ and 531Ad differ in the $\mathrm{hmgA}$ gene mutation and 23 mutations, most of which locate to intergenic regions and insertion sequences.

Vibrio anguillarum is the causative agent of vibriosis, a devastating disease characterized by terminal hemorrhagic septicemia that affects numerous fresh and salt-water fish species ${ }^{1,2}$. Among the hosts that $V$. anguillarum infects are species of enormous economic importance such as salmon, turbot, rainbow trout, sea bass, sea bream, cod, eel, and ayu ${ }^{1}$. Although never recognized as a human pathogen, one case of fatal infection in an immunocompromised patient has been documented ${ }^{3}$. Studies on the molecular mechanisms of pathogenicity in this bacterium led to the identification of various virulence factors ${ }^{1,4,5}$ such as motility $^{6-9}$, adhesion ${ }^{9}$, the lipopolysaccharide ${ }^{1}$, outer membrane proteins ${ }^{1}$, DNase, protease, and hemolysin activities ${ }^{4,10-13}$, as well as efficient iron uptake systems ${ }^{14-17}$. V. anguillarum also possesses quorum sensing systems involved in regulation of expression of virulence factors ${ }^{18,19}$. The pJM1-type plasmid-mediated anguibactin iron-uptake system is one of the best characterized virulence factors present in numerous serotype $\mathrm{O} 1 \mathrm{~V}$. anguillarum strains ${ }^{14,15,20,21}$. It consists of the low-molecular-weight siderophore anguibactin and a receptor complex that facilitates transport of ferric-anguibactin across the membranes ${ }^{22,23}$. The vast majority of the pJM1-type plasmids include genes that code for enzymes that participate in the synthesis of anguibactin, outer membrane and periplasmic components of the ferric-anguibactin receptor complex, and regulatory elements ${ }^{24-27}$. Studies on V. anguillarum 531A, a strain isolated from a diseased fish on the Atlantic coast, showed that it carries a pJM1-like plasmid, pJHC1, and can grow in the presence of higher concentrations of iron chelators when compared to other strains like the well-studied strain $775^{28,29}$. Another unique and intriguing property of $V$. anguillarum $531 \mathrm{~A}$ is that it secretes very high amounts of a brown pigment. We aimed to understand the genetics and biochemistry of this property.

Center for Applied Biotechnology Studies, Department of Biological Science, California State University Fullerton, Fullerton, CA, USA. Correspondence and requests for materials should be addressed to M.E.T. (email: mtolmasky@ fullerton.edu) 

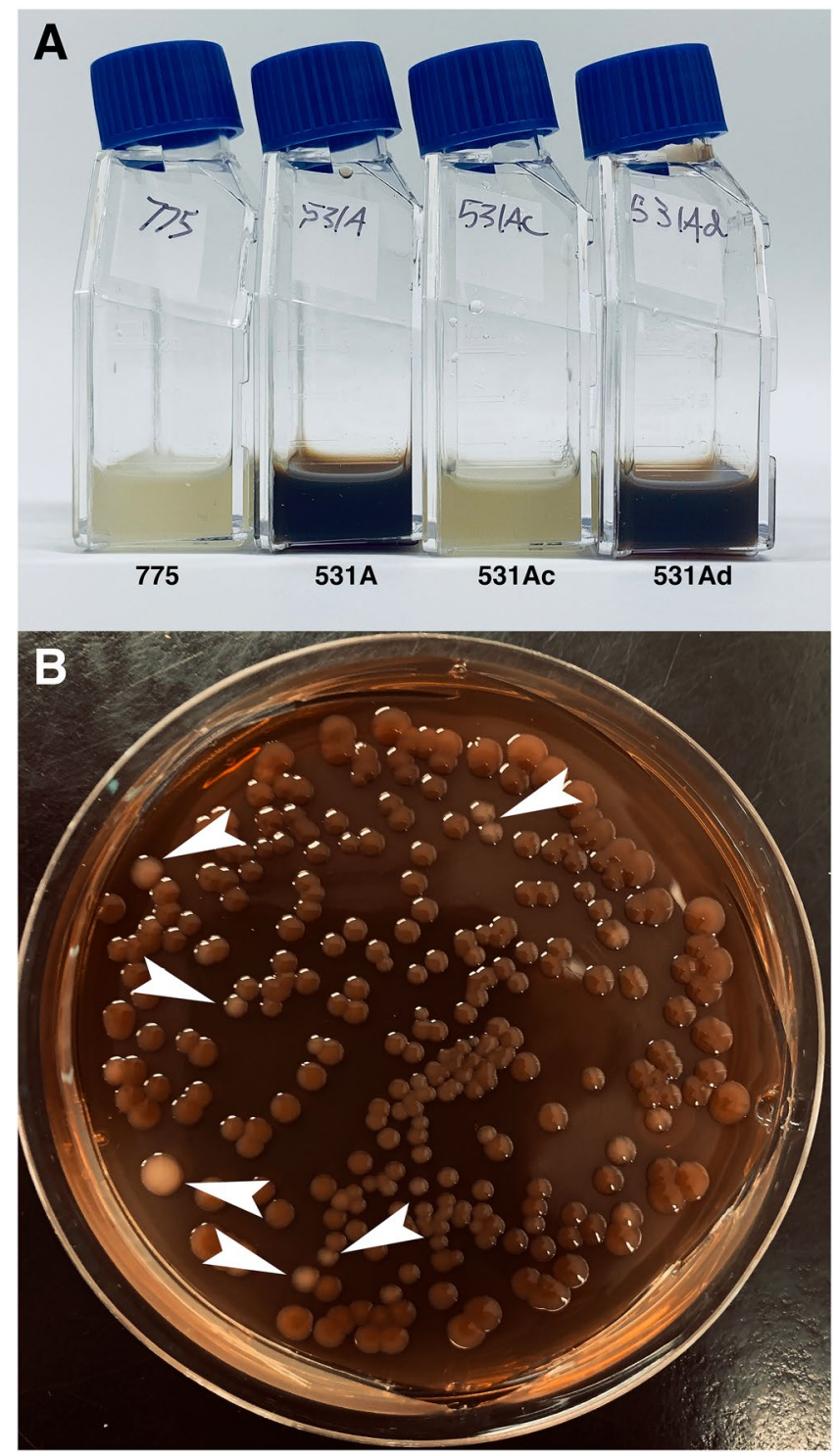

Figure 1. Pigment production in V. anguillarum strains. (A) Cultures were carried out in TSBS at $25^{\circ} \mathrm{C}$ for $72 \mathrm{~h}$ with shaking. (B) V. anguillarum $531 \mathrm{~A}$ was plated in TSAS and incubated at $25^{\circ} \mathrm{C}$ for $48 \mathrm{~h}$. The white arrowheads show some of the clear colonies. Variants 531Ac and 531Ad were isolated from a similar plate.

In this work, we report that V.anguillarum 531A is a mix of two variants, one of them producing dark brown colonies due to production of an ochronotic-like pigment that is correlated with a DNA duplication that disrupts the $h m g A$ gene. The lack of a functional homogentisate 1,2-dioxygenase leads to accumulation of homogentisate in the tyrosine degradation pathway. Numerous reports described bacteria that produce the ochronotic pigment pyomelanin when homogentisate accumulates and undergoes oxidation and polymerization ${ }^{30-38}$.

\section{Results}

V. anguillarum 531A, a strain isolated from diseased Oncorhynchus kisutch in the Atlantic Ocean, carries the pJM1-type plasmid pJHC1 and produces higher levels of anguibactin than the strain $775^{28,29,39}$. While performing assays to characterize $V$. anguillarum 531A, we noticed that, unlike 775 and all other V. anguillarum strains in our collection, it produces a dark brown pigment (Fig. 1A). Another observation was that upon plating, the isolate produced two kinds of colonies, about $95 \%$ of them were dark and the rest with the usual clear color (Fig. 1B). A representative of each of the colonies was isolated, and the new strains were named 531Ad (dark) and 531Ac (clear). These strains conserved their pigmentation characteristics when they were subsequently cultured in TSBS or TSAS (Fig. 1A).

To determine if $V$. anguillarum 531Ac and 531Ad were two independent strains or one derives from the other we did whole-genome sequencing. The draft sequences of strains 531 AC and 531AD had a total of 2,039,599 and 2.204.992 high-quality paired-end reads, respectively; $99.9 \%$ of the reads were mapped, resulting in mean nucleotide coverage of 30X for both genomes. The corrected reads showed an average length of 128 (531Ac) and 

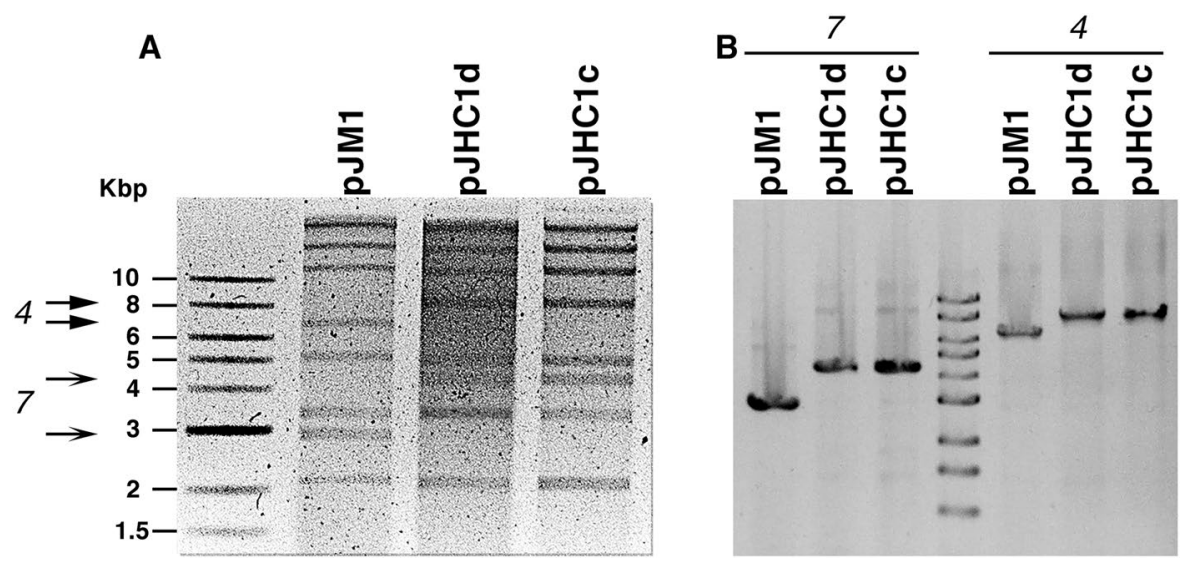

Figure 2. Restriction endonuclease digestion of V. anguillarum plasmids. Ethidium bromide-stained $0.7 \%$ agarose gel electrophoresis of BamHI digestions of plasmids pJM1, pJHJC1d, and pJHC1c. Original pictures of both gels are shown in the Supplementary Information.

123 (531Ad) bp and there were 119 (531Ac) and 124 (531Ad) contigs larger than $500 \mathrm{bp}$. The draft genomes have an $\mathrm{N}_{50}$ contig size of 96,636 (531Ac) and 100,331 (531Ad) bp with a maximum contig length of 347,928 (531Ac) and 321,926 (531Ad). The GC content (\%) of the genomes was determined as $44.3 \%$, a value consistent with previous measurements using other $V$. anguillarum strains ${ }^{40}$. The genomic differences between both strains were evaluated by performing genome comparisons. Two-way average nucleotide identity (ANI) calculation and the tetra nucleotide correlation analysis among both isolates showed a 99,9\% value and a score 1.0, respectively. Also, tetra nucleotide correlation against all the database of JSpeciesWS, showed the highest score against $V$. anguillarum 775 . These results strongly suggest that $V$. anguillarum $531 \mathrm{Ac}$ and $531 \mathrm{Ad}$ could be considered to be the essentially same strain with a small number of nucleotide differences. To validate these results, a core-genome phylogeny of $V$. anguillarum 531Ac and 531Ad and V. anguillarum genomes from GenBank. The core-genome contains 1538 genes and the pan-genome contains 13,038 genes. The phylogenetic relation among the $V$. anguillarum $531 \mathrm{Ac}$ and 531Ad genomes as determined by core-genome phylogeny analysis showed that the pairwise distance is 0.000344655 substitution per $100 \mathrm{bp}$, which indicates the presence of a few SNPs between both isolates (Supplementary Fig. S1 and Table S1). Mutation prediction among both isolates showed that besides the duplication of of the $\mathrm{hmgA}$ gene described below, there are 23 mutations among the V.anguillarum $531 \mathrm{Ac}$ and 531Ad genomes. While most mutations were found within intergenic regions and insertion sequence (ISVa5 and ISVa2) (Supplementary Table S2), one of them occurred within the TyrP (tyrosine-specific transport protein) coding sequence. This mutation resulted in a Q349P amino acid change (Supplementary Table S2) within the transmembrane domain. However, prediction of transmembrane helices using the TMHMM software showed that the mutation does not induce significant structural differences. As a consequence, it is most probable that the one amino acid difference does not affect tyrosine uptake. Future research will permit to confirm this observation. A final confirmation of the identity between $V$. anguillarum 531 Ac and 531 Ad was carried out by restriction analysis of their plasmid content. V. anguillarum strains usually harbor pJM1-type plasmids whose DNA sequences slightly differ ${ }^{14,28,41,42}$. Therefore, identical restriction digestion patterns of both pJM1-type plasmids would be a strong indication that one of the strains derives from the other. The plasmid originally isolated from $V$. anguillarum 531A was called pJHC1, then the plasmids isolated from the variants 531 Ac and 531Ad were called pJHC1c and pJHC1d. Figure 2A shows a comparison of the products of BamHI restriction digestion of pJHC1c and pJHC1d, as well as the prototype plasmid pJM1. This figure shows that both strains, 531Ac and 531Ad, shared the same signature pJM1-type plasmid, the BamHI fragments 4 and 7 showed identical molecular size in pJHC1c and $\mathrm{pJHC1d}$, and were larger than those from pJM1. The correspondence between the fragments was confirmed by PCR using as primers oligonucleotides at the edges of the BamHI fragments 4 and 7 based on the pJM1 nucleotide sequence (Fig. 2B). These results agree with the genomic analyses and indicates that both variants have a common origin.

Production of the ochronotic type pigment pyomelanin is caused by the accumulation of homogentisate formed by the action of 4-hydroxyphenylpyruvate dioxygenase, coded for by the $h p p D$ gene, on 4-hydroxyphenylpyruvic acid in the tyrosine metabolic pathway (Fig. 3) $33,34,43,44$. Accumulation of homogentisate can occur when homogentisate 1,2-dioxygenase, the enzyme encoded by $h m g A$ that metabolizes this compound to 4-maleylacetoacetate, is not present (Fig. 3). The homogentisate is oxidized and polymerized to produce pyomelanin ${ }^{30,34}$. Figure 3 shows the tyrosine metabolic pathway underscoring the steps relevant to formation of pyomelanin. To determine if this pathway is responsible for the hyperproduction of the brown pigment in $V$. anguillarum 531Ad, we cloned the chromosome I region encompassing the $h p p D$ and $h m g A$ genes from strains 531 Ac and 531Ad. Introduction of the recombinant clones pP531 Ac and pP531Ad into E. coli resulted in clear and dark colonies, respectively (Fig. 3). Inspection of the nucleotide sequences of both regions cloned showed that there was a 410-bp tandem repeat within the nucleotide sequence of the V. anguillarum 531Ad hmgA gene $\left(h m g A^{*}\right)$ that results in a frameshift and truncation of the product of translation. This modification leaves 156 out of a total of 375 amino acids in the complete protein (Fig. 3 and Supplementary Fig. S2). To confirm that 


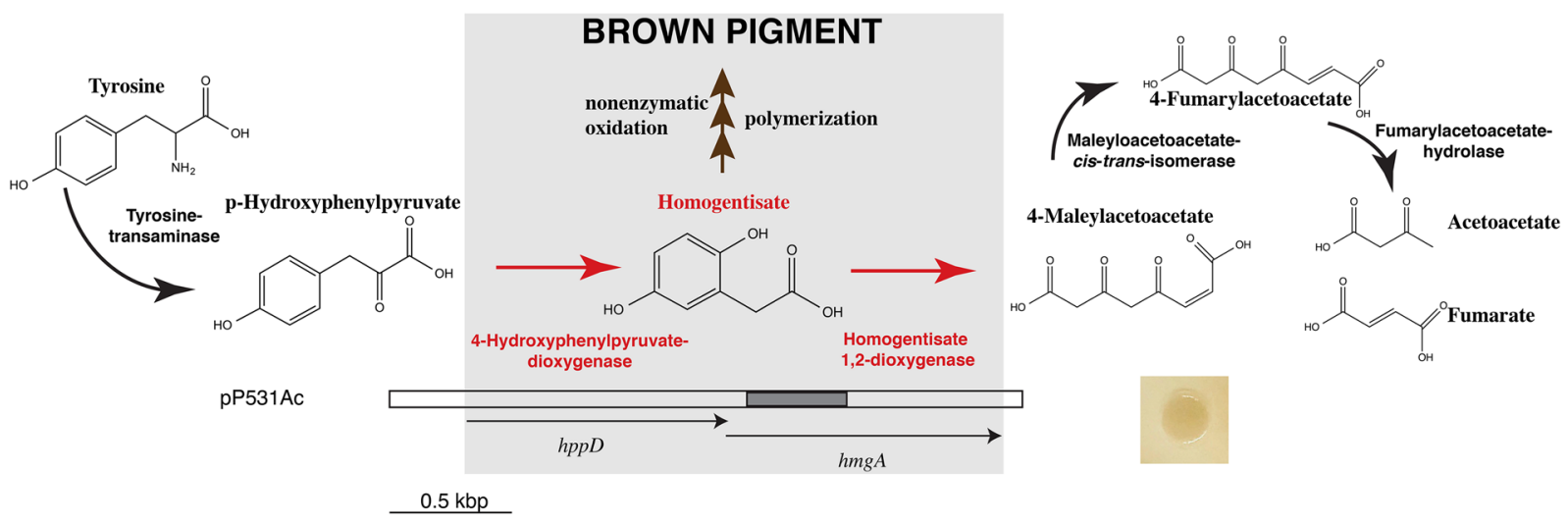

pP531Ad
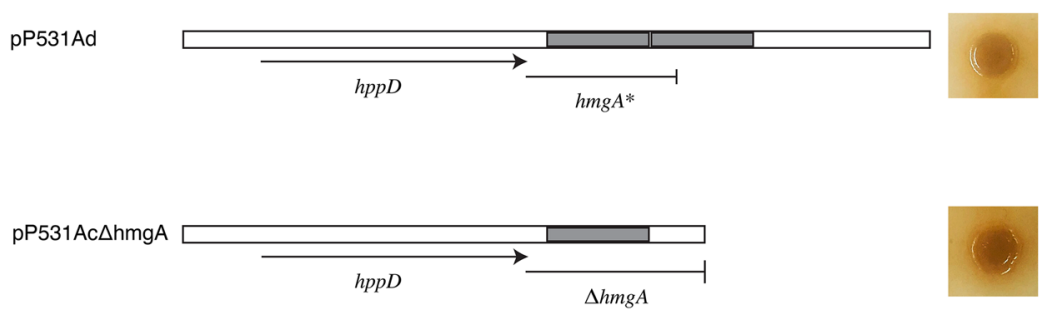

Figure 3. Pathway for tyrosine metabolism and production of the brown pigment. The top portion of the figure shows the tyrosine catabolic pathway and the key enzymes for formation of the brown pigment are shown within the gray box. The box also includes the region of DNA that includes the $h p p D$ and $h m g A$ genes. The lower portion of the figure shows diagrams of the fragments and genes included in all three recombinant plasmids. The grayed region is directly repeated in the 531Ad strain; $h m g A^{*}$ indicates that the 410-bp tandem duplication (highlighted in gray) in this gene produces a sequence that codes for a truncated homogentisate 1,2-dioxygenase (Supplementary Fig. S2). The phenotypes produced by each recombinant plasmid in E. coli cultured in LB agar are shown to the right of the genetic maps.

the lack of a functional homogentisate 1,2-dioxygenase was responsible for the high production of pigment, the region cloned in pP531Ac was used as template to generate a DNA fragment that includes the complete $h p p D$ and only a portion of the $h m g A$ gene. This fragment was used to generate the recombinant clone pP531Ac $\Delta \mathrm{hmgA}$ (Fig. 3 and Supplementary Fig. S2). As expected, E. coli cells carrying this recombinant plasmid produced highly pigmented colonies (Fig. 3). Next, a recombinant clone harboring the $h m g A$ gene under the control of the T7 promoter was generated inserting the appropriate DNA segment from strain 531Ac into pACYC177, a vector compatible with pCR-Blunt II-TOPO. This plasmid, pPhmgA531Ac, was introduced into E. coli BL21(DE3) already harboring pP531Ac $\Delta \mathrm{hmgA}$, which produces brown colonies (Fig. 4). The transformant strain, E. coli BL21(DE3) (pP531Ac $\Delta$ hmgA, pPhmgA531Ac), produced clear colonies further confirming that lack of a functional $h m g A$ gene is a direct cause of production of the brown pigment (Fig. 4). The experiments described in this paragraph led us to conclude that $V$. anguillarum 531Ad produces high amounts of pyomelanin due to the lack of a functional homogentisate 1,2-dioxygenase. Inspection of all 60 complete V. anguillarum genomes in GenBank indicated that all of them include a gene coding for a full homogentisate 1,2-dioxygenase. Therefore, 531Ad is the only $V$. anguillarum strain described to date that harbors a disrupted $h m g A$ gene.

It had been observed that $V$. anguillarum $531 \mathrm{~A}$ can grow at higher concentrations of iron chelators when compared to $V$. anguillarum 775 due to higher production of anguibactin ${ }^{28,29}$. Since V. anguillarum $531 \mathrm{~A}$ is a mix of two variants, 531 Ac and 531Ad, it is possible that only one of them possesses the ability to grow at higher concentrations of iron chelators. The minimal inhibitory concentrations of EDDHA for both variants were $20 \mu \mathrm{M}$ while the value for strain 775 was $10 \mu \mathrm{M}$. This result indicated that the presence or absence of an intact copy of the $h m g A$ gene does not impact the iron proficiency of $V$. anguillarum in the experimental conditions.

Coculturing assays were used to compare the fitness of strains 531Ad and 531Ac to determine if the ability to produce high amounts of pyomelanin in high quantities was advantageous when growing in TSBS culture medium. A 1:1 mixture of the strains was cultured, and the relative frequencies were determined every 20 generations. Up to 60 generations, the ratio $531 \mathrm{Ad} / 531 \mathrm{Ac}$ was approximately 1 indicating that both strains were equally fit when growing in TSBS.

Since a recent report described a variant of the Aeromonas salmonicida 4-hydroxyphenylpyruvate dioxygenase that is thermolabile due to the amino acids S18, P103, and L119, which in thermostable variants are mostly T18, Q103, and P11937, we identified these important amino acids within the V. anguillarum version of the enzyme. A comparison of both sequences showed that except for a short stretch at the $\mathrm{N}$-terminus, the amino acid sequences shared $76 \%$ identity and $85 \%$ similarity (Fig. 5). Furthermore, the amino acids at the key positions for thermolability in the V. anguillarum enzyme were T, Q, and $\mathrm{P}$ as it is the case of those that are thermostable (Fig. 5). 


\section{E. coli BL21(DE3) pP531Ac $\Delta$ hmgA}

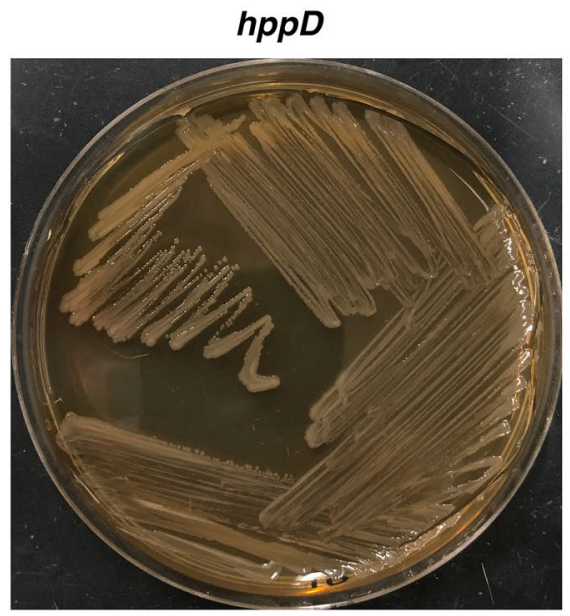

\section{E. coli BL21(DE3) \\ pP531Ac $\Delta$ hmgA \\ pPhmgA531Ac}

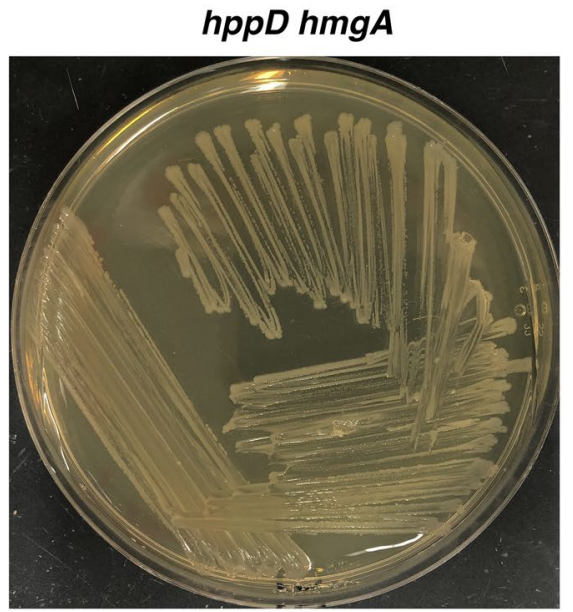

Figure 4. $\mathrm{h} m g A$ gene complementation. E. coli BL21(DE3) (pP531Ac $\Delta \mathrm{hmgA}$ ) and E. coli BL21(DE3)

(pP531Ac $\Delta \mathrm{hmgA}$, pPhmgA531Ac) were plated on Trypticase soy agar with the addition of $1 \%(\mathrm{w} / \mathrm{v}) \mathrm{NaCl}$.

Va

As

Va

As

Va

As

Va

As

Va

As

$\mathrm{Va}$

As

\section{6}

NPLGTDGFEFVEYTAADPAGIAQLKELFTSLGFAEIAKHRSKEAWLYRQGDINFIVNSQP NPLG+DGFEFVEYTA D GIA LK LF SLGFAE+AKH+ K+ WLYRQGDINF+VN++P

14 NPLGSDGFEFVEYTAPDAKGIAALKALFVSLGFAEVAKHKHKQCWLYRQGDINEVVNAEP

HSQAAAFAKVHGP SVCGMAFRVHDASVAMQHVQANGGREYKTQIGPMELS I PAIYGIGDS HSQA FA +HGPSVCGMAFRV DA A + $\mathrm{G}+++\mathrm{IG} \mathrm{MEL}+\mathrm{IPAIYGIG}+\mathrm{S}$ HSQAEQFASLHGPSVCGMAFRVADAGKAQPYAITKGAKPFVGKIGIMELNIPAIYGIGES L FVDRYG + SIYDVDF FY D RM + + GL+EIDHLTHNV++G+MD+WS FYER 134 TLSFVDRYGDKGSIYDVDFVFYPDWQTRMAEVDAGLYEIDHLTHNVQRGNMDVWSNFYER 185 IGNFREIRYFDIEGKLTGLVSRAMTAPCGKIRIPINESSDDKSQIEEFIREYNGEGIQHI IGNFREIRYFDIEGKLTGL SRAMTAPCGKIRIPINESSDDKSQIEE++R+Y GEGIQHI 194 IGNFREIRYFDIEGKLTGLHSRAMTAPCGKIRIPINESSDDKSQIEEYLRQYKGEGIQHI 245 ALSTDDIYQTVRTLRERGMDFMPTPDTYYEKVSDRVEGHQEDVDQLKALRILIDGAPMKD AL++ DIYQT+RTLR RG FMPTPDTYYEKV+ RVEGHQED+ LK L+ILIDG+P KD 254 ALASSDIYQTIRTLRGRGTGFMPTPDTYYEKVNSRVEGHQEDLASLKDLQILIDGSPTKD

Figure 5. 4-Hydroxyphenylpyruvate-dioxygenase amino acid sequences comparison. Alignment of the amino acid sequences of the 4-hydroxyphenylpyruvate-dioxygenase proteins from V. anguillarum (Va) and $A$. salmonicida (As) (accession number MH909233.1). Amino acids involved in thermolability are highlighted in green. 


\section{Discussion}

The production of the ochronotic pigment pyomelanin has been reported in numerous organisms including humans, where the accumulation of homogentisate produces the syndrome known as ochronosis or alkaptonuria $^{45,46}$. Numerous bacteria have been identified as producers of a pyomelanin-type pigment, which occurs through the metabolic pathway of tyrosine degradation. Homogentisate is produced by the action of 4-hydroxyphenylpyruvate dioxygenase on 4-hydroxyphenylpyruvic acid. When this metabolite accumulates, it undergoes oxidation and polymerization producing the brown pigment pyomelanin. This process has been identified as the cause behind the production of the pigment in several bacteria such as V. anguillarum ${ }^{33}, V$. cholerae $^{38}$, A. salmonicida ${ }^{37}$, Legionella pneumophila ${ }^{31}$, Shewanella colwelliana ${ }^{32}$, Burkholderia cepacia ${ }^{35}$, B. cenocepacia $^{36}$, Streptomyces avermitilis ${ }^{34}$, Ralstonia solanacearum ${ }^{30}$. Previous studies identified VanT and RpoS as positive regulators of pyomelanin production by enhancing expression of 4-hydroxyphenylpyruvate dioxygenase in $V$. anguillarum cultures entering stationary phase $\mathrm{e}^{33,44}$. However, based on the published information ${ }^{33,44}$, these levels of pigment production must be much lower than those observed in strain 531Ad, in which the lack of homogentisate 1,2-dioxygenase activity leads to accumulation of the pyomelanin precursor and production of unusually high amounts of the pigment. The results obtained by searching the GenBank database indicating that all sequenced $V$. anguillarum strains show a complete $h m g A$ gene confirms that the mechanism of production of pyomelanin in strain 531Ad is, to our best knowledge, exceptional among this species. A search for this characteristic, i.e., higher pigmentation, in other bacteria showed that a subset of Burkholderia cepacia strains includes a deficient $h m g A$ gene. In this case, rather than the truncation, the variant of the $h m g A$ gene codes for a protein that has an amino acid substitution that is detrimental for enzymatic activity ${ }^{35}$.

Since we did not know the basis of the appearance of two variants in the sample kept at $-80^{\circ} \mathrm{C}$ in the laboratory collection, a battery of experiments was carried out to determine of both V. anguillarum 531Ac and 531 Ad have a common origin and also if there is a fitness difference in regular laboratory growth conditions. Whole-genome sequencing and plasmid restriction enzyme digestion analyses showed that both variants are essentially the same strain with the exception of the $h m g A$ gene and other minor mutations. Furthermore, comparison of their iron uptake proficiency also failed to show any difference. Of course, it is possible that the production of pyomelanin is advantageous in particular conditions not tested in this work. For example, it was recently found in comparisons of different Klebsiella pneumoniae strains, which showed that a dominant Klebsiella pneumoniae strain in the host does not show enhanced fitness in the mixed growth assay ${ }^{47}$. Several studies found pyomelanin functions that could enhance fitness in bacteria. Examples of these functions are protection against oxidative stress $^{30,36,48}$ or enhancement of bacterial virulence ${ }^{35,38}$. A $V$. cholerae mutant obtained by mini- Tn5 insertion within $h m g A$ overproduced pyomelanin, expressed more toxin-coregulated pilus and cholera toxin, and showed enhanced colonization of intestines in an infant mice model of infection ${ }^{38}$. On the other hand, other reports show a reduction in virulence of bacteria that develop the capability to produce the pigment ${ }^{49}$. The information available seems to indicate that high production of pyomelanin is correlated with pleiotropic changes that may differ among bacteria. Future experiments will clarify our understanding of the possible role of pyomelanin in high quantities in the physiology and virulence of $V$. anguillarum.

\section{Methods}

Bacterial strains, plasmids, primers, and media. The relevant characteristics of bacterial strains and plasmids used in this work are described in Table 1. V. anguillarum 531A was isolated from a diseased fish in the coast of Maine ${ }^{28,29,39}$. V. anguillarum 775 is a prototype serotype $\mathrm{O} 1$ strain; it was used for comparison of growth under iron deficiency conditions and as a source of plasmid pJM1 ${ }^{22}$. Escherichia coli TOP10 (Life Technologies) and BL21(DE3 $)^{50}$ were used as hosts of recombinant clones. Plasmids pCR-Blunt II-TOPO (Life Technologies) and pACYC $177^{51}$ were used as cloning vehicles. V. anguillarum strains were cultured at $25^{\circ} \mathrm{C}$ in Trypticase soy broth with the addition of $1 \%(\mathrm{w} / \mathrm{v}) \mathrm{NaCl}$ (TSBS), and for cultures in solid medium the medium was supplemented with $2 \%$ agar (TSAS) $)^{42}$. E. coli strains were cultured at $37^{\circ} \mathrm{C}$ in LB broth or LB agar (2\%). Oligonucleotides to be used as primers or for DNA sequencing were purchased from Integrated DNA Technologies or BioSynthesis Inc.

General procedures. Plasmid DNA isolation and PCR reactions were carried out using the QIAspin miniprep (QIAGEN) and the Invitrogen Platinum SuperFi PCR Master Mix (ThermoFisher Scientific) kits, respectively. Primers used to amplify DNA fragments are listed in Table 2. Restriction endonuclease and T4 DNA ligase treatments were performed according to the supplier's recommendations (New England Biolabs). Transformation of competent E. coli strains was carried out as described by the supplier (Life Technologies). Briefly, $50 \mu \mathrm{l}$ of the competent cells suspension were thawed on ice before adding the DNA sample. The cells were then incubated for 30 minutes on ice, followed by a heat shock at $42^{\circ} \mathrm{C}$ for 30 seconds. Then $250 \mu \mathrm{l}$ of LB broth were added, and the cells were incubated for 1 hour at $37^{\circ} \mathrm{C}$ with shaking. The cells were then plated on LB agar containing $1 \mathrm{mg} / \mathrm{ml}$ ampicillin. Amino acid sequence comparisons were carried out using the BLAST tool at the National Center for Biotechnology information ${ }^{52}$.

Determination of minimal inhibitory concentration (MIC) of ethylenediamine di(o-hydroxyphenyl) acetic acid (EDDHA). MIC of the synthetic iron chelator of the iron chelator EDDHA was assessed culturing the strains in M9 minimal medium containing incremental EDDHA concentrations up to $40 \mu \mathrm{M}^{22,28}$.

Fitness assays. Fitness of V. anguillarum strains 531Ac and 531Ad were compared coculturing a 1:1 mixture of the strains and determining their ratio every 20 generations as described previously ${ }^{53,54}$. 


\begin{tabular}{|c|c|c|}
\hline Bacteria or plasmids & Relevant characteristics or genotype & Source or reference \\
\hline \multicolumn{3}{|l|}{ Bacteria } \\
\hline V. anguillarum $531 \mathrm{~A}$ & Isolate from an infected fish. Carries pJHC1, a pJM1-like plasmid & Tolmasky et al. $^{29}$ \\
\hline V. anguillarum 531Ad & High pigment producer & This work \\
\hline V. anguillarum $531 \mathrm{Ac}$ & Low, or no, pigment producer & This work \\
\hline E. coli TOP10 & 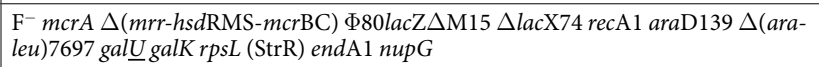 & Life Technologies \\
\hline E. coli BL21(DE3) & $\mathrm{F}^{-}$ompT hsdSB $\left(r B^{-} m B^{-}\right)$gal dcm (DE3) & Studier \& Moffatt, $1986^{50}$ \\
\hline \multicolumn{3}{|l|}{ Plasmids } \\
\hline pCR-Blunt II-TOPO & Cloning vector $\mathrm{KAN}^{\mathrm{r}} \mathrm{ZEO}^{\mathrm{r}}$ & Life Technologies \\
\hline pACYC177 & Cloning vector $\mathrm{KAN}^{\mathrm{r}} \mathrm{AMP}^{\mathrm{r}}$ & Rose, $1988^{51}$ \\
\hline pP531Ac & $h p p D$ - $h m g A$ fragment from strain $531 \mathrm{Ac}$ cloned into pCR-Blunt II-TOPO & This work \\
\hline pP531Ad & $h p p D-h m g A *$ fragment from strain 531Ad cloned into pCR-Blunt II-TOPO & This work \\
\hline pP531Ac $\Delta$ hmgA & $h p p D-\Delta h m g A$ fragment from strain 531Ac cloned into pCR-Blunt II-TOPO & This work \\
\hline pPhmgA531Ac & $h m g A$ fragment from strain 531Ac cloned into pACYC177 & This work \\
\hline
\end{tabular}

Table 1. Bacterial strains and plasmids used in this study.

\begin{tabular}{|c|c|c|}
\hline Primer name & Sequence & Coordinates $^{\mathrm{a}}$ \\
\hline $\mathrm{F}^{\mathrm{a}}$ & AGGCAGTGTCGGTTTTTAATG & $1-21$ \\
\hline $\mathrm{R}^{\mathrm{a}}$ & TAACCCATCTCTTGTGCCGT & $2586-2567$ \\
\hline $\mathrm{R} 2^{\mathrm{a}}$ & CCACGGAAAGATCACCGTGCC & $2093-2073$ \\
\hline FhmgAT7 ${ }^{\mathrm{a}, \mathrm{b}}$ & ATCGATGCGAAATTAATACGACTCACTATAGGGGAGATCATTCAACGCAAAGGCAATC & $1288-1311$ \\
\hline $\mathrm{R} h m g A \mathrm{~T} 7^{\mathrm{a}, \mathrm{b}}$ & $\underline{\text { AAGCTTAACCCATCTCTTGTGCCGTTTTTTAG }}$ & $2586-2560$ \\
\hline BamHI4Fc & GAATAGATCAGCTGCTTTGGGATCC & $34731-34755$ \\
\hline BamHI4R ${ }^{c}$ & ATCCCAATGTTTCAATGTACGAGGATCC & $41395-41368$ \\
\hline BamHI7Fc & GATCCTCTTTTCGTTCAGCACTGG & $55321-55344$ \\
\hline $\mathrm{BamHI}^{\mathrm{n}} \mathrm{R}^{\mathrm{c}}$ & GGCGTGGTGTGCTTTCGGATC & $58207-58187$ \\
\hline
\end{tabular}

Table 2. Primers. ${ }^{a}$ Coordinates according to the Vibrio anguillarum 775 chromosome I sequence, accession number CP002284, region: $1733068.1737000 .{ }^{b}$ Double underlined sequence is the T7 promoter. Single underlined and italics underlined are the ClaI and HindIII restriction sites, respectively. ${ }^{c}$ Coordinates according to the Vibrio anguillarum 775 pJM1 plasmid, accession number AY312585.

Pigment production. V. anguillarum strains were inoculated in TSBS or plated on solid media and cultured at $25^{\circ} \mathrm{C}$ for $36 \mathrm{~h}$. E. coli strains were plated on $\mathrm{LB}$ agar and incubated for $36 \mathrm{~h}$ at $37^{\circ} \mathrm{C}$. The production of pigment was readily visible.

Genome sequencing and bioinformatic analysis. Genomic DNA was extracted using the Master Pure DNA purification kit following the manufacturer's instructions (Epicentre, Madison, WI, USA). Libraries were generated using the Illumina Nextera kit (Illumina Inc., San Diego, CA) as described by Baym et al..$^{55}$, and sequenced with the Illumina NextSeq500 equipment at the Microbial Genome Sequencing Center (Pittsburgh, PA). De novo assembly was performed with SPADES assembler, version 3.3.1 ${ }^{56}$. The annotation was performed using the RAST server ${ }^{57}$ and the predictions were confirmed using the BLAST software ${ }^{52}$. The quality of the sequences and assembly were evaluated using FASTQC software (https://www.bioinformatics.babraham.ac.uk/ projects/fastqc/) and the QUAST software ${ }^{58}$, respectively. The Average Nucleotide Identity and pairwise tetra nucleotide correlation were performed using JSpeciesWS online service ${ }^{59}$. The mutation prediction between both isolates was performed using breseq ${ }^{60}$. The core genome prediction was carried out using the Roary software ${ }^{61}$ including seventy $V$. anguillarum genomes from GenBank. The core-genome phylogeny was performed using RaxML ${ }^{62}$ and substitution model was predicted with JModelTest $2^{63}$. The default Bootstrap test was used to evaluate branch supports. The tree representation was done using FigTree (https://github.com/rambaut/figtree/). The transmembrane domain prediction was done using the TMHMM software (http://www.cbs.dtu.dk/services/ $\mathrm{TMHMM} /$ ).

\section{Data Availability}

Published accession numbers of sequences used in this study are included in the article. The nucleotide sequences of specific regions from strains 531Ac and 531Ad have been provided GenBank accession numbers MK791314 and MK791315. Whole genome sequences of V. anguillarum 531 Ac and 531 Ad provided GenBank accession numbers VSLE00000000 and VSLF00000000, respectively. Bacterial strains are available upon request. 


\section{References}

1. Frans, I. et al. Vibrio anguillarum as a fish pathogen: virulence factors, diagnosis and prevention. J. Fish Dis. 34, 643-661, https://doi. org/10.1111/j.1365-2761.2011.01279.x (2011).

2. Actis, L. A., Tolmasky, M. E. \& Crosa, J. H. Vibriosis. In Fish Diseases and Disorders, Volume 3: Viral, Bacterial and Fungal Infections (eds Woo, P. T. K. \& Bruno, D. W.) 523-557 (CAB International, 1999).

3. Sinatra, J. A. \& Colby, K. Notes from the field: fatal Vibrio anguillarum infection in an immunocompromised patient - Maine, 2017. MMWR Morb. Mortal. Wkly. Rep. 67, 962-963, https://doi.org/10.15585/mmwr.mm6734a5 (2018).

4. Gao, X. et al. Survival, virulent characteristics, and transcriptomic analyses of the pathogenic Vibrio anguillarum under starvation stress. Front Cell Infect Microbiol 8, 389, https://doi.org/10.3389/fcimb.2018.00389 (2018).

5. Holm, K. O., Nilsson, K., Hjerde, E., Willassen, N. P. \& Milton, D. L. Complete genome sequence of Vibrio anguillarum strain NB10, a virulent isolate from the gulf of Bothnia. Stand Genomic Sci 10, 60, https://doi.org/10.1186/s40793-015-0060-7 (2015).

6. McGee, K., Horstedt, P. \& Milton, D. L. Identification and characterization of additional flagellin genes from Vibrio anguillarum. J. Bacteriol. 178, 5188-5198 (1996).

7. Milton, D. L., O'Toole, R., Horstedt, P. \& Wolf-Watz, H. Flagellin A is essential for the virulence of Vibrio anguillarum. J. Bacteriol. 178, 1310-1319 (1996).

8. O’Toole, R., Milton, D. L. \& Wolf-Watz, H. Chemotactic motility is required for invasion of the host by the fish pathogen Vibrio anguillarum. Mol. Microbiol. 19, 625-637 (1996).

9. Ormonde, P., Horstedt, P., O’Toole, R. \& Milton, D. L. Role of motility in adherence to and invasion of a fish cell line by Vibrio anguillarum. J. Bacteriol. 182, 2326-2328 (2000).

10. Crisafi, F. et al. $\mathrm{NaCl}$ concentration in the medium modulates the secretion of active EmpA protease in Vibrio anguillarum at posttranscriptional level. J. Appl. Microbiol. 119, 1494-1501, https://doi.org/10.1111/jam.12957 (2015).

11. Rodkhum, C. et al. Putative virulence-related genes in Vibrio anguillarum identified by random genome sequencing. J. Fish Dis. 29, 157-166, https://doi.org/10.1111/j.1365-2761.2006.00692.x (2006).

12. Mou, X., Spinard, E. J., Driscoll, M. V., Zhao, W. \& Nelson, D. R. H-NS is a negative regulator of the two hemolysin/cytotoxin gene clusters in Vibrio anguillarum. Infect. Immun. 81, 3566-3576, https://doi.org/10.1128/IAI.00506-13 (2013).

13. Crosa, J. H. Signal transduction and transcriptional and posttranscriptional control of iron-regulated genes in bacteria. Microbiol. Mol. Biol. Rev. 61, 319-336 (1997).

14. Di Lorenzo, M. \& Stork, M. Plasmid-encoded iron uptake systems. Microbiology spectrum 2, https://doi.org/10.1128/microbiolspec. PLAS-0030-2014 (2014).

15. Li, Y. \& Ma, Q. Iron acquisition strategies of Vibrio anguillarum. Frontiers in cellular and infection microbiology 7, 342, https://doi. org/10.3389/fcimb.2017.00342 (2017).

16. Tolmasky, M. E. \& Crosa, J. H. Regulation of plasmid-mediated iron transport and virulence in Vibrio anguillarum. Biol. Met. 4, 33-35 (1991).

17. Balado, M. et al. The siderophore piscibactin Is a relevant virulence factor for Vibrio anguillarum favored at low temperatures. Front. Microbiol. 9, 1766, https://doi.org/10.3389/fmicb.2018.01766 (2018).

18. Li, X., Yang, Q., Dierckens, K., Milton, D. L. \& Defoirdt, T. RpoS and indole signaling control the virulence of Vibrio anguillarum towards gnotobiotic sea bass (Dicentrarchus labrax) larvae. PLoS One 9, e111801, https://doi.org/10.1371/journal.pone.0111801 (2014).

19. Tang, L. et al. Expression, secretion and bactericidal activity of type VI secretion system in Vibrio anguillarum. Arch. Microbiol. 198, 751-760, https://doi.org/10.1007/s00203-016-1236-2 (2016).

20. Chen, Q., Actis, L. A., Tolmasky, M. E. \& Crosa, J. H. Chromosome-mediated 2,3-dihydroxybenzoic acid is a precursor in the biosynthesis of the plasmid-mediated siderophore anguibactin in Vibrio anguillarum. J. Bacteriol. 176, 4226-4234, https://doi. org/10.1128/jb.176.14.4226-4234.1994 (1994).

21. Di Lorenzo, M. et al. Complete sequence of virulence plasmid pJM1 from the marine fish pathogen Vibrio anguillarum strain 775 . J. Bacteriol. 185, 5822-5830 (2003).

22. Tolmasky, M. E. \& Crosa, J. H. Molecular cloning and expression of genetic determinants for the iron uptake system mediated by the Vibrio anguillarum plasmid pJM1. J. Bacteriol. 160, 860-866 (1984).

23. Actis, L. A. et al. Characterization of anguibactin, a novel siderophore from Vibrio anguillarum 775(pJM1). J. Bacteriol. 167, 57-65 (1986).

24. Salinas, P. C., Tolmasky, M. E. \& Crosa, J. H. Regulation of the iron uptake system in Vibrio anguillarum: evidence for a cooperative effect between two transcriptional activators. Proc. Natl. Acad. Sci. USA 86, 3529-3533 (1989).

25. Waldbeser, L. S., Tolmasky, M. E., Actis, L. A. \& Crosa, J. H. Mechanisms for negative regulation by iron of the fatA outer membrane protein gene expression in Vibrio anguillarum 775. J. Biol. Chem. 268, 10433-10439 (1993).

26. Tolmasky, M. E., Actis, L. A. \& Crosa, J. H. Genetic analysis of the iron uptake region of the Vibrio anguillarum plasmid pJM1: molecular cloning of genetic determinants encoding a novel trans activator of siderophore biosynthesis. J. Bacteriol. 170, 1913-1919 (1988).

27. Koster, W. L., Actis, L. A., Waldbeser, L. S., Tolmasky, M. E. \& Crosa, J. H. Molecular characterization of the iron transport system mediated by the pJM1 plasmid in Vibrio anguillarum 775. J. Biol. Chem. 266, 23829-23833 (1991).

28. Tolmasky, M. E., Salinas, P. C., Actis, L. A. \& Crosa, J. H. Increased production of the siderophore anguibactin mediated by pJM1like plasmids in Vibrio anguillarum. Infect. Immun. 56, 1608-1614 (1988).

29. Tolmasky, M. E., Actis, L. A. \& Crosa, J. H. A single amino acid change in AngR, a protein encoded by pJM1-like virulence plasmids, results in hyperproduction of anguibactin. Infect. Immun. 61, 3228-3233 (1993).

30. Ahmad, S. et al. Genetic determinants for pyomelanin production and its protective effect against oxidative stress in Ralstonia solanacearum. PLoS One 11, e0160845, https://doi.org/10.1371/journal.pone.0160845 (2016).

31. Aubi, O. et al. Discovery of a specific inhibitor of pyomelanin synthesis in Legionella pneumophila. J. Med. Chem. 58, 8402-8412, https://doi.org/10.1021/acs.jmedchem.5b01589 (2015).

32. Coon, S. L. et al. Homogentisic acid is the product of MelA, which mediates melanogenesis in the marine bacterium Shewanella colwelliana D. Appl. Environ. Microbiol. 60, 3006-3010 (1994).

33. Croxatto, A. et al. VanT, a homologue of Vibrio harveyi LuxR, regulates serine, metalloprotease, pigment, and biofilm production in Vibrio anguillarum. J. Bacteriol. 184, 1617-1629 (2002).

34. Denoya, C. D., Skinner, D. D. \& Morgenstern, M. R. A Streptomyces avermitilis gene encoding a 4-hydroxyphenylpyruvic acid dioxygenase-like protein that directs the production of homogentisic acid and an ochronotic pigment in Escherichia coli. $J$. Bacteriol. 176, 5312-5319 (1994).

35. Gonyar, L. A., Fankhauser, S. C. \& Goldberg, J. B. Single amino acid substitution in homogentisate 1,2-dioxygenase is responsible for pigmentation in a subset of Burkholderia cepacia complex isolates. Environ. Microbiol. Rep. 7, 180-187, https://doi.org/10.1111/17582229.12217 (2015).

36. Keith, K. E., Killip, L., He, P., Moran, G. R. \& Valvano, M. A. Burkholderia cenocepacia C5424 produces a pigment with antioxidant properties using a homogentisate intermediate. J. Bacteriol. 189, 9057-9065, https://doi.org/10.1128/JB.00436-07 (2007).

37. Qiao, Y. et al. 4-Hydroxyphenylpyruvate dioxygenase thermolability is responsible for temperature-dependent melanogenesis in Aeromonas salmonicida subsp. salmonicida. Appl. Environ. Microbiol. 85, https://doi.org/10.1128/AEM.01926-18 (2019). 
38. Valeru, S. P. et al. Role of melanin pigment in expression of Vibrio cholerae virulence factors. Infect. Immun. 77, 935-942, https://doi. org/10.1128/IAI.00929-08 (2009).

39. Strout, R., Sawyer, E. \& Coutermarsh, B. Pathogenic vibrios in confinement-reared and feral fishes of the Maine-New Hampshire coast. J. Fish. Res. Board Can. 35, 403-408 (1978).

40. Castillo, D. et al. Comparative genome analyses of Vibrio anguillarum strains reveal a link with pathogenicity traits. $m$ Systems $\mathbf{2}$, https://doi.org/10.1128/mSystems.00001-17 (2017).

41. Ronneseth, A. et al. Comparative assessment of Vibrio virulence in marine fish larvae. J. Fish Dis. 40, 1373-1385, https://doi. org/10.1111/jfd.12612 (2017)

42. Tolmasky, M. E., Actis, L. A., Toranzo, A. E., Barja, J. L. \& Crosa, J. H. Plasmids mediating iron uptake in Vibrio anguillarum strains isolated from turbot in Spain. J. Gen. Microbiol. 131, 1989-1997, https://doi.org/10.1099/00221287-131-8-1989 (1985).

43. Yang, H. et al. The tyrosine degradation gene $h p p D$ is transcriptionally activated by HpdA and repressed by HpdR in Streptomyces coelicolor, while $h p d A$ is negatively autoregulated and repressed by HpdR. Mol. Microbiol. 65, 1064-1077, https://doi.org/10.1111/ j.1365-2958.2007.05848.x (2007).

44. Weber, B., Croxatto, A., Chen, C. \& Milton, D. L. RpoS induces expression of the Vibrio anguillarum quorum-sensing regulator VanT. Microbiology 154, 767-780, https://doi.org/10.1099/mic.0.2007/014167-0 (2008).

45. Roberts, N. B., Curtis, S. A., Milan, A. M. \& Ranganath, L. R. The pigment in alkaptonuria relationship to melanin and other coloured substances: a review of metabolism, composition and chemical analysis. JIMD Rep. 24, 51-66, https://doi. org/10.1007/8904_2015_453(2015).

46. Gil, J. A., Wawrzynski, J. \& Waryasz, G. R. Orthopedic manifestations of ochronosis: pathophysiology, presentation, diagnosis, and management. Am. J. Med. 129(536), e531-536, https://doi.org/10.1016/j.amjmed.2016.01.010 (2016).

47. Agard, M. J., Ozer, E. A., Morris, A. R., Piseaux, R. \& Hauser, A. R. A genomic approach to identify Klebsiella pneumoniae and Acinetobacter baumannii strains with enhanced competitive fitness in the lungs during multi-strain neumonia. Infect. Immun., https://doi.org/10.1128/IAI.00871-18 (2019).

48. Turick, C. E., Caccavo, F. Jr. \& Tisa, L. S. Pyomelanin is produced by Shewanella algae BrY and affected by exogenous iron. Can. J. Microbiol. 54, 334-339, https://doi.org/10.1139/w08-014 (2008).

49. Wang, Z. et al. Vibrio campbellii hmgA-mediated pyomelanization impairs quorum sensing. virulence, and cellular fitness. Front. Microbiol. 4, 379, https://doi.org/10.3389/fmicb.2013.00379 (2013).

50. Studier, F. W. \& Moffatt, B. A. Use of bacteriophage T7 RNA polymerase to direct selective high-level expression of cloned genes. J. Mol. Biol. 189, 113-130 (1986).

51. Rose, R. E. The nucleotide sequence of pACYC177. Nucleic Acids Res. 16, 356 (1988).

52. Altschul, S. F., Gish, W., Miller, W., Myers, E. W. \& Lipman, D. J. Basic local alignment search tool. J. Mol. Biol. 215, 403-410, https:// doi.org/10.1016/S0022-2836(05)80360-2 (1990).

53. Bui, D. Y. et al. Differences in resolution of $m w r$-containing plasmid dimers mediated by the Klebsiella pneumoniae and Escherichia coli XerC recombinases: Potential implications in dissemination of antibiotic resistance genes. J. Bacteriol. 188, 2812-2820, https:/l doi.org/10.1128/Jb.188.8.2812-2820.2006 (2006).

54. Capiaux, H., Lesterlin, C., Perals, K., Louarn, J. M. \& Cornet, F. A dual role for the FtsK protein in Escherichia coli chromosome segregation. Embo Reports 3, 532-536, https://doi.org/10.1093/embo-reports/kvf116 (2002).

55. Baym, M. et al. Inexpensive multiplexed library preparation for megabase-sized genomes. PLoS One 10, e0128036, https://doi. org/10.1371/journal.pone.0128036 (2015).

56. Bankevich, A. et al. SPAdes: a new genome assembly algorithm and its applications to single-cell sequencing. J. Comput. Biol. 19, 455-477, https://doi.org/10.1089/cmb.2012.0021 (2012).

57. Aziz, R. K. et al. The RAST Server: rapid annotations using subsystems technology. BMC Genomics 9, 75, https://doi. org/10.1186/1471-2164-9-75 (2008).

58. Gurevich, A., Saveliev, V., Vyahhi, N. \& Tesler, G. QUAST: quality assessment tool for genome assemblies. Bioinformatics 29, 1072-1075, https://doi.org/10.1093/bioinformatics/btt086 (2013).

59. Richter, M., Rossello-Mora, R., Oliver Glockner, F. \& Peplies, J. JSpeciesWS: a web server for prokaryotic species circumscription based on pairwise genome comparison. Bioinformatics 32, 929-931, https://doi.org/10.1093/bioinformatics/btv681 (2016).

60. Deatherage, D. E. \& Barrick, J. E. Identification of mutations in laboratory-evolved microbes from next-generation sequencing data using breseq. Methods Mol. Biol. 1151, 165-188, https://doi.org/10.1007/978-1-4939-0554-6_12 (2014).

61. Page, A. J. et al. Roary: rapid large-scale prokaryote pan genome analysis. Bioinformatics 31, 3691-3693, https://doi.org/10.1093/ bioinformatics/btv421 (2015)

62. Stamatakis, A. RAxML version 8: a tool for phylogenetic analysis and post-analysis of large phylogenies. Bioinformatics 30, 1312-1313, https://doi.org/10.1093/bioinformatics/btu033 (2014).

63. Darriba, D., Taboada, G. L., Doallo, R. \& Posada, D. jModelTest 2: more models, new heuristics and parallel computing. Nat Methods 9, 772, https://doi.org/10.1038/nmeth.2109 (2012).

\section{Acknowledgements}

This work was supported by Public Health Service grants 2R15AI047115 (M.E.T.) from the National Institute of Allergy and Infectious Diseases, National Institutes of Health, SC3GM125556 (M.S.R.) from the National Institute of General Medical Sciences, National Institutes of Health, and California State University Fullerton.

\section{Author Contributions}

M.E.T. conceived the study, designed, and carried out the early experiments. M.E.T., M.S.R., J.F. and V.B. designed experiments. V.B., J.F., B.F., J.S., K.L.Q., K.P., E.F., A.R., K.V., F.C., A.J.F., K.B., J.Z., V.L., K.D., G.T. and K.R. performed experiments. M.E.T. wrote the first draft of the manuscript, all other authors contributed to editing.

Additional Information

Supplementary information accompanies this paper at https://doi.org/10.1038/s41598-019-51126-8.

Competing Interests: The authors declare no competing interests.

Publisher's note Springer Nature remains neutral with regard to jurisdictional claims in published maps and institutional affiliations. 
(c) (i) Open Access This article is licensed under a Creative Commons Attribution 4.0 International License, which permits use, sharing, adaptation, distribution and reproduction in any medium or format, as long as you give appropriate credit to the original author(s) and the source, provide a link to the Creative Commons license, and indicate if changes were made. The images or other third party material in this article are included in the article's Creative Commons license, unless indicated otherwise in a credit line to the material. If material is not included in the article's Creative Commons license and your intended use is not permitted by statutory regulation or exceeds the permitted use, you will need to obtain permission directly from the copyright holder. To view a copy of this license, visit http://creativecommons.org/licenses/by/4.0/.

(C) The Author(s) 2019 